\title{
A Bolometer Bridge for Standardizing Radio-Frequency Voltmeters
}

\author{
By Myron C. Selby and Lewis F. Behrent
}

\begin{abstract}
In the course of work on r-f standardization projects of the National Bureau of Standards, practical equipment and techniques were developed for measurement of 20 millivolts to 1.9 volts at radio frequencies below 700 megacycles, with further broadening of these ranges still in progress. The devices employed do not require frequency corrections, so that results are based on direct-current measurements, which can be made quite accurately. Reproducibility and agreement, with independent methods was found to be \pm 1 percent or better. The primary function of the equipment is to make available a standard voltage of any desired value and frequency within the above range that would be required for a highly accurate and at the same time practical voltmeter-calibration procedure. In addition, the equipment may be used for direct measurement of $r$-f generator voltages; as a known standard of $\mathrm{r}-\mathrm{f}$ impedance; and for accurate power measurements of 20 microwatts to 100 milliwatts. An analytical study of the range-limiting factors of this technique is presented, appropriate curves and illustrations are shown, and major steps of procedure are listed.
\end{abstract}

An independent method and appropriately designed equipment for accurate $r$-f voltage determinations based on measurements of direct current and of linear dimensions along a transmission line of known characteristic impedance are also described.

\section{Introduction}

In searching for voltage standardization methods, at frequencies from $10 \mathrm{kc}$ to $300 \mathrm{Mc},{ }^{1}$ promising results were obtained in the early part of 1946 by using the bolometer bridge. This eventually led to the development of the equipment and technique described herein. To meet requirements the methods and equipment were to be practical, reliable, of high precision, and were to approach an accuracy of 1 percent. Reliability was to be assured by cross-checking results of two or more independent methods. Individual methods and techniques were to be adapted for regular use on the basis of accuracy, speed of measurement, and of their individual maximum ranges of voltage and frequency.

In the light of present experience, measurements at radio frequencies to accuracies of 1 percent

\footnotetext{
1 M. C. Selby, High-frequency voltage measurements, NBS Circular C481. Central Radio Propagation Laboratory Report CRPL-8-2 issued A pril 14, 1948.
}

were considered of high precision. Reproducibility of results, as well as agreement between individual primary methods, was to be within \pm 1 percent, or better. Frequency errors were to be negligible. Herein "precision" is used in reference to sensitivity, incidental variations, scalereading facilities, and other errors of observation; "accuracy" refers to the true value of the quantity measured. Results may thus be precise and not accurate, but not vice versa, i. e., once a value is stated to be accurate to a certain degree it is necessary that measurements be precise to the same degree. The term "systematic error" is frequently used in place of "accuracy" as defined here, and "accidental error" is used in place of "precision."

Among the devices suitable for primary measurements of $r-f$ voltages in the sense stated above, the most practical operating principles employed were: the bolometer-bridge using the substitution of $\mathrm{r}-\mathrm{f}$ for $\mathrm{d}-\mathrm{c}$ power, measurement of current 
through a known resistance, cathode-ray beam deflection, and the electrometer. Each of these principles is useful over a considerable range of voltage and frequency. The bolometer bridge method and the extent of its agreement with other methods will be described here.

\section{Basic Principle}

The principle of $\mathrm{r}-\mathrm{f}$ power measurement by means of a bolometer bridge is widely known. It consists briefly of balancing a d-c wheatstone bridge, having a bolometer (a device the resistance of which is a function of the power dissipated in it) in one of its arms, and of rebalancing it again after some of the d-c power in the bolometer is replaced by $\mathrm{r}-\mathrm{f}$ power. Under these two conditions the difference in the $d$-c power consumed by the bridge gives a precise indication of $\mathrm{r}-\mathrm{f}$ power applied. An elementary circuit diagram of such a bridge is shown in figure 1. As used here, the term "bolometer" refers to r-f power detectors having resistance elements of high temperature coefficient of resistivity.

The fundamental difference between $r-f$ power bolometer-bridges and those for r-f voltage rests in the fact that the reactive component of the bolometer impedance has no great bearing on the

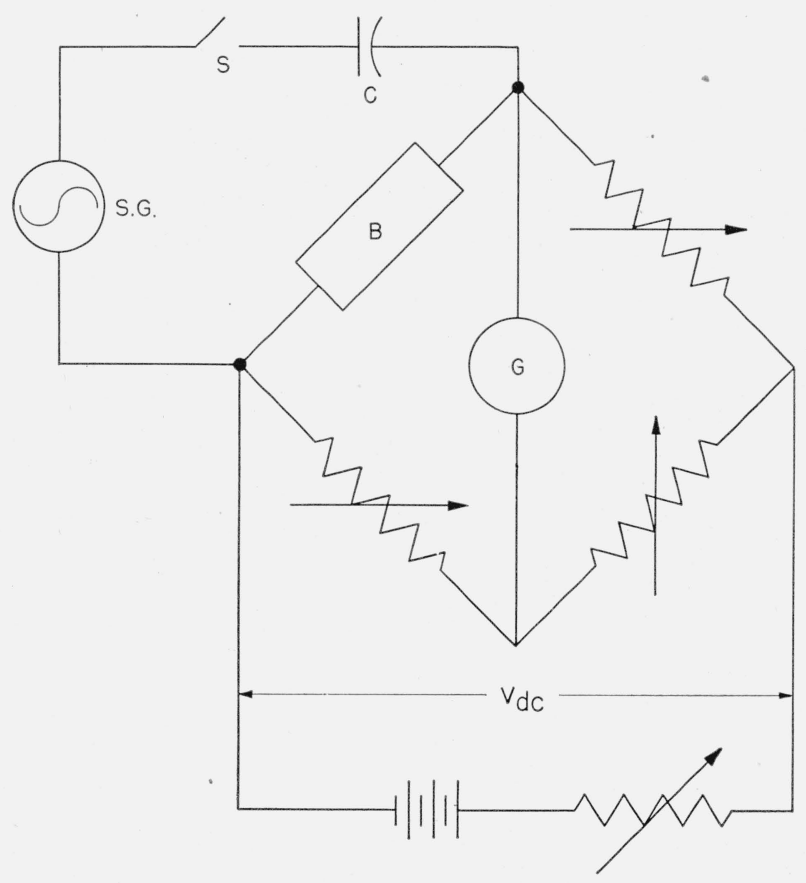

Figure 1. Elementary circuit diagram of a bolomete bridge. accuracy of the power measured, whereas it is of prime importance for voltage measurements. For the present purpose, the bolometer impedance configuration must meet the condition that the $\mathrm{d}-\mathrm{c}$ voltage across it be practically equal to the rms value at all frequencies considered. This condition can be met only if the bolometer admittance consists essentially of a pure conductance shunted by any value of positive or negative susceptance. Appropriate types of bolometers were therefore chosen, and a mounting for them was designed to meet the above condition over a wide frequency range. The equivalent circuit of the assembly is shown in figure 2. It is evident that $X_{T}=\omega L_{T}$

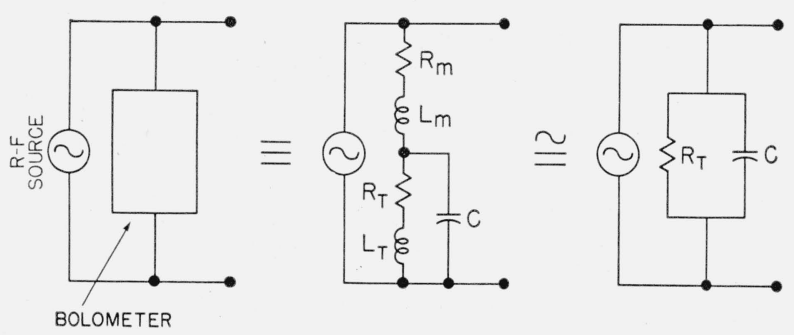

Figure 2. Equivalent circuit of a bolometer assembly applicable for $r$ - $f$ voltage measurements.

$R_{m}$ and $L_{m}$, the incidental series resistance and inductance of the bolometer mount and $L_{T}$, the series inductance of the bolometer ate assumed to be negligible. $\quad R_{T}$ is the bolometer resistance.

and $X_{m}\left(=\omega L_{m}\right)$ must be negligible, although the value of $C$ is of no direct significance except when the bolometer assembly is used for power output measurements, in which case it may be tuned out. $R_{m}$ may have a finite value, but it should be negligible as compared with $R_{T}$ because of its variation with frequency as a result of skin effect.

\section{Technique and Its Limitations}

\section{Bolometers and Bridge Circuity}

The choice of the type of bolometer and the design of its mount seemed to be the most critical features in establishing $r-f$ voltages of known value. Two types were available for consideration, namely, the thermistor and the wollaston-wire (or "little-fuse") unit. A glance at figure 3 will show that the physical dimensions of the former were more adaptable for the purpose, whereas the series inductance and size of the available wollaston-wire units limited their usefulness only to relatively low frequencies. Other advantages of the thermistor are indicated below. 


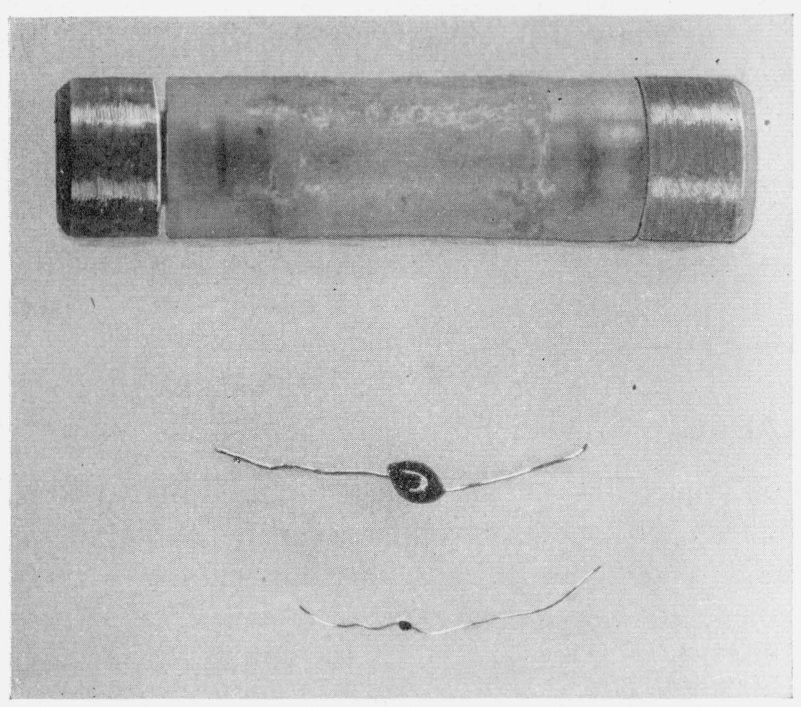

Figure 3. Two thermistor beads and a barretter in its mount.

A thermistor ${ }^{2}$ is a semiconductor such as uranium oxide $\left(\mathrm{U}_{3} \mathrm{O}_{8}\right)$, or a mixture of nickel oxide (NiO) and manganese oxide $\left(\mathrm{Mn}_{2} \mathrm{O}_{3}\right)$, having a large negative resistance-temperature coefficient. The beads used in this work are glass-coated and differ in diameter depending on characteristics desired. The smallest applied in this case had an approximate over-all outside diameter of $0.015 \mathrm{in}$. with platinum connecting-lead diameter of 0.001 in. and a single lead length of several thousandths in. A wollaston-wire is a platinum wire of the order of $0.0004 \mathrm{in}$. in diameter drawn inside a silver wire; this silver coat is removed over a small section by etching with a solution of nitric acid $\left(\mathrm{HNO}_{3}\right)$; the exposed platinum core constitutes the active section of the bolometer and may be 0.1 in. long. With mounting provisions the final over-all dimensions are usually greater than those of thermistors and consequently prove less desirable for some applications. Greater overload handling capacity is a major factor in favor of the thermistor. The power sensitivity of a commercial unit wollaston-wire type bolometer (such as the Sperry type barretter $)^{3}$ is approximately 5 $\mathrm{ohms} / \mathrm{mw}$ (for 200 -ohm initial condition at $25^{\circ} \mathrm{C}$ ). A corresponding figure for the unmounted thermistor mentioned is approximately $15 \mathrm{ohms} / \mathrm{mw}$. It will be shown later that the thermistor sensi-

\footnotetext{
${ }^{2}$ J. A. Becker, C. B. Green, and G. L. Pearson, Properties and uses of thermistors - thermally sensitive resistors. Trans. AIEE 65, No. 11, p. 711 (Nov. 1946).

${ }^{3}$ Sperry Gyroscope Co., Great Neck, N. Y., Model 821 barretter.
}

tivity is reduced when mounted close to bodies having good heat conductivity.

Schematic circuit diagrams of a single-and twothermistor bridge are shown in figures 4 and 5 . The single thermistor bridge (fig. 4) requires chokes to keep r-f current out of the d-c circuit, whereas in the two-thermistor bridge (fig. 5) the d-c circuit has no r-f potential across it, thus eliminating the need for chokes.

The general expression for the rms voltage $V$, for the two-thermistor bridge, as derived in appendix I, is :

$$
\begin{aligned}
V= & \frac{R_{T}}{\left(R_{T}+R_{b}\right)(1+\alpha)} \\
& {\left[\alpha\left(V_{R 2}-V_{R 1}\right)\left(2 V_{0}-V_{R 2}-V_{R 1}\right)\right]^{\frac{1}{2}}, }
\end{aligned}
$$

where

$\alpha=R_{T 1} / R_{T 2}=$ constant ratio of the individual thermistor resistances when the bridge is balanced with or without r-f applied to it. $R_{T}=R_{T 1}+R_{T 2} ; R_{T 1}, R_{T 2}$, and $R_{b}$ are as shown in figure 5 .

$V_{R 1}=$ voltage drop across the resistor in series with the battery at initial d-c bridge balance (switch $S$ open).

$V_{R 2}=$ voltage drop across that resistor at second balance (switch $S$ closed).

$V_{0}=$ battery voltage, which is assumed to remain constant with load variation.

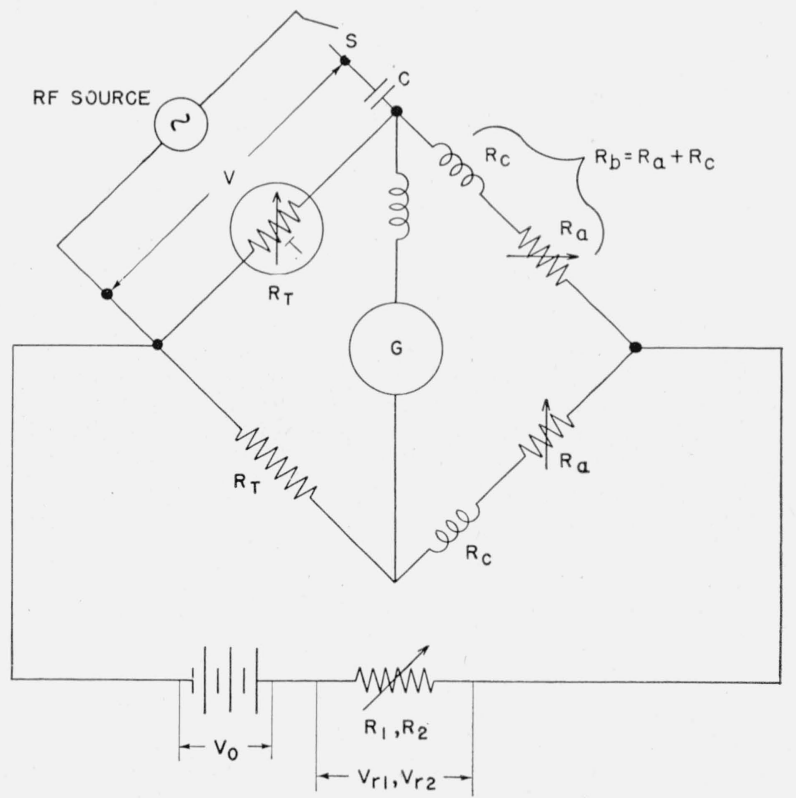

Figure 4. Schematic diagram of a single thermistor bridge. $R_{T}=$ thermistor resistance; $R_{a}=$ resistance-ratio arms; $R_{c}=$ resistance of $\mathrm{r} \cdot \mathrm{f}$ chokes in bridge arms. 


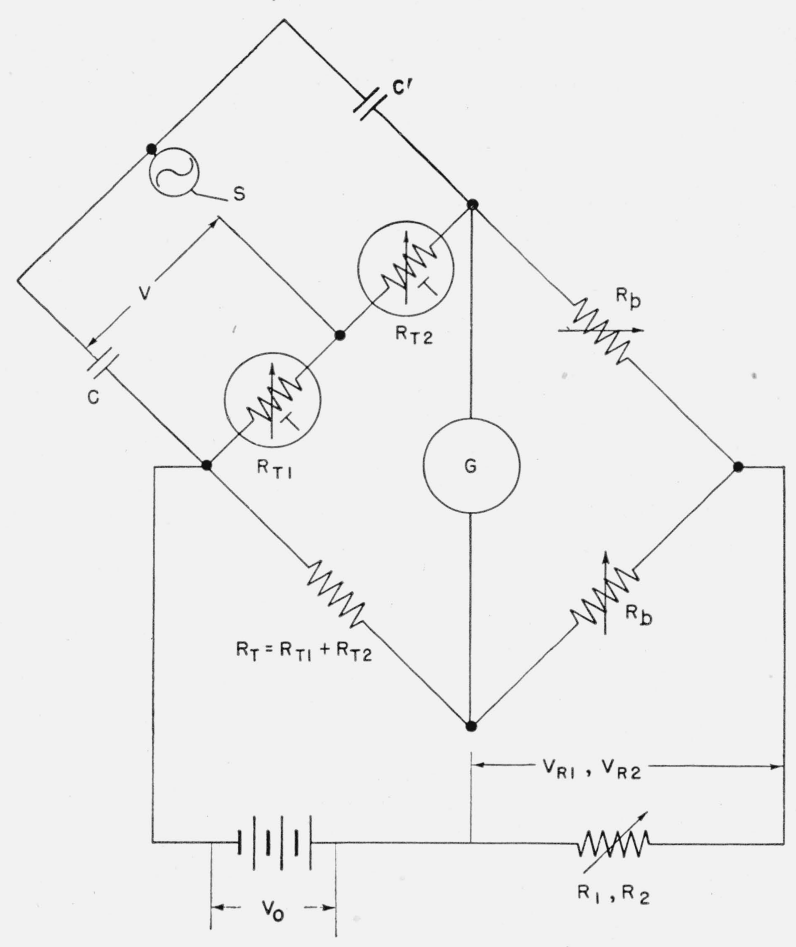

Figure 5. Schematic diagram of two-thermistor bridge. $R_{T_{1}}$ and $R_{T_{2}}$ are individual thermistor resistances.

Condensers $C$ and $C^{\prime}$ prevent direct current flowing to or from the r-f source or device connected to the measurement terminals. $C$ and $C^{\prime}$ are chosen large enough so that the effective load presented to the $r-f$ source is essentially the $d-c$ resistance of the two thermistors connected in parallel.

For the single-thermistor bridge, and for the two-thermistor bridge when $R_{T 1} \cong R_{T^{*}}$ the r-f voltage is:

$$
V=\frac{R_{T}}{2\left(R_{T}+R_{b}\right)}\left[\left(V_{R 2}-V_{R 1}\right)\left(2 V_{0}-V_{R 2}-V_{R 1}\right)\right]^{\frac{1}{2}} .
$$

$\mathrm{R}$-f chokes in the single-thermistor bridge reduce errors caused by incidental rectification of $\mathrm{r}-\mathrm{f}$ voltages entering the $\mathrm{d}-\mathrm{c}$ circuit. They also reduce the loading effect of the bridge on the r-f source. A choke in series with each of the $R_{b}$ arms is shown in figure 4. This simplifies operation and computation when the chokes are matched. The chokes must be shielded, properly installed, and must have stable resistance and a sufficiently high impedance over the entire frequency range of application. Difficulties in meeting these requirements make the two-thermistor bridge preferable.
Equation 2 is further simplified when $R_{1}$ can be made negligibly small as compared with $R_{2}$. In this case:

$$
V=\frac{R_{T}}{2\left(R_{T}+R_{b}\right)}\left[V_{R 2}\left(2 V_{0}-V_{R 2}\right)\right]^{\frac{1}{2}} .
$$

If, in addition, $R_{b}=R_{T}$, the simplest case of an equal-arm bridge is obtained and

$$
V=\frac{1}{4}\left[V_{R 2}\left(2 V_{0}-V_{R 2}\right)\right]^{\frac{1}{2}} .
$$

Typical thermistor characteristic curves (see footnote 2), one of which is illustrated in figure 15 , show that there are two possible values of thermistor current for each value of voltage across it over part of the range. The voltage maximum will be referred to as the "turning point." In order to assure operation over the high temperature coefficient portion of the curve (low $R_{T}$ values), $V_{0}$ must be equal to or larger than the turning-point voltage. If $V_{0}$ is lower than the turning-point voltage, other means, such as application of external heat, of $r-f$ or a-f power, may be used to obtain an initial bridge balance at the desired $R_{T}$ value. However, this procedure is unsatisfactory, because $R_{T}$ may assume a high value during the process of rebalancing the bridge for a measurement; this instability may necessitate several time-consuming balancing attempts. Another difficulty may be encountered with parallel operation of thermistors as a result of the presence of two possible values of thermistor resistance for every value of applied voltage. It is conceivable (and this was observed in practice) that one of the two thermistors will operate in its high-resistance region, whereas the other will be in its low-resistance region and will therefore carry most of the r-f current. This may take place when the thermistors have widely different values of turning-point voltages and resistance-temperature coefficients. It can be avoided by approximately matching the thermistors and by choosing operating values reasonably removed from the turning point. The reader may at this point wonder how it is at all possible to obtain stable operation with negative temperature-coefficient resistors connected in parallel to the same power source, because a slight decrease in the resistance of one of them would cause consecutive reactions decreasing its resistance further, and reducing the current in the other shunt ele- 
ments. The answer is that the above reaction may in fact take place up to a certain equilibrium point at which the voltage drop in the source impedance reduces the voltage applied to the thermistors to a value necessary to maintain the thermistor resistances constant. The difference in operation of negative temperature-resistancecoefficient elements as against zero or positive temperature-resistance units is that the former cannot be indiscriminately connected to a power source of insufficient internal (or series external current-limiting) impedance without damaging either the load or the power source, or both.

As will be shown later, batteries are preferable to rectified a-c power sources for wide voltagerange and accuracy. Once batteries are chosen, $V_{0}$ cannot be readily varied except in steps of single cells. This feature makes it difficult to use an equal-arm bridge at a negligible value of $R_{1}$. One might mention here the desirability of having $R_{T}$ as low as possible. A lower $R_{T}$ corresponds to a higher thermistor temperature with a lower consequent interference from ambient temperature variations.

The error in $V$ as a result of thermistor mismatch (i. e., the error caused by assuming $\alpha=1$ ) is given by

$$
\frac{\Delta V}{V}=1-2 \alpha^{\frac{1}{2}}(1+\alpha)^{-1}
$$

Appendix II gives the derivation of this error, and figure 6 shows its magnitude versus $\alpha$. Here, and throughout the discussion below, $\Delta$ designates a relatively small finite increment of a quantity. There is, however, no difficulty in measuring $\alpha$, as will be shown in the discussion of the final equipment. It may be seen from figure 6 that a mismatch of about 5 percent can be safely neglected, because the error in $V$ will thereby not exceed 0.1 percent. Equation 1 must therefore be used for values of $\alpha$ larger than 1.05, whereas for values of $\alpha$ closer to 1, eq 3 (or its equivalent when $R_{1} \neq 0$ ) is sufficient. Factors contributing individually an error of 0.1 percent in $V$ may be neglected on the assumption that the total accumulated error will not exceed 1 percent.

The accuracy with which $\alpha$ must be determined, to not exceed a 0.1 -percent error in $V$, is derived in appendix III and is shown in figure 6. A lower value of $\alpha$ requires less accuracy in its determination.

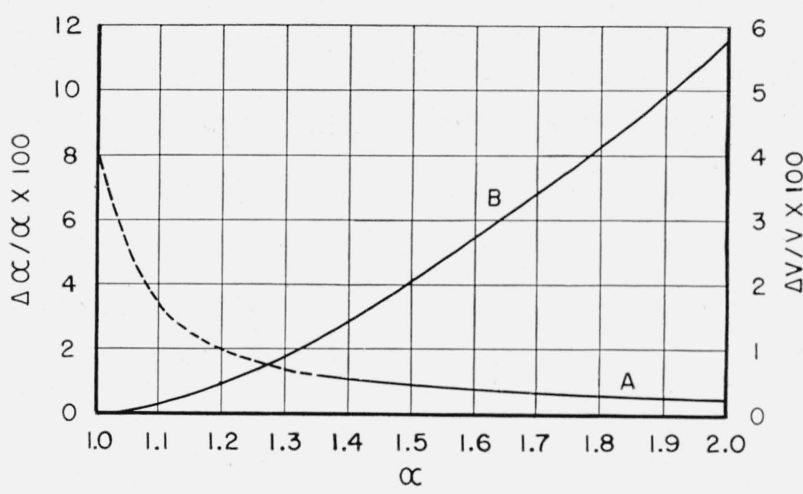

Figure 6. (A) Permissible error in determining $\alpha$ for a 0.1-percent error in the $r$-f voltage, $V$. Broken-line section represents eq 27, appendix $I I I$. Broken line,

$$
\frac{\Delta \alpha}{\alpha}=\frac{-\left(2 \sigma-1.002 \sigma^{2}\right) \pm \sqrt{\left(2 \sigma-1.002 \sigma^{2}\right)^{2}+0.008 \sigma^{2}}}{2} .
$$

Solid line, $\frac{\Delta \alpha}{\alpha}=\frac{2(1+\alpha)}{1-\alpha} 10^{-3}$. (B) Error in V when mismatch between the thermistors of a two-thermistor bridge is neglected. $\frac{\Delta V}{V}=\left(1-\frac{2 \sqrt{\alpha}}{1+\alpha}\right)$.

It is assumed throughout the text that $\alpha$ remains constant for all values of $d-c$ and $r-f$ voltages within the working limits of the bridge. Experimental evidence indicated this to be essentially true for the great majority of individual pairs of thermistors of the type used when their lead lengths and position in the gap of the mount were closely alike. The variation of $\alpha$ in these cases did not exceed 1.1 and the agreement with independent methods was well within \pm 1 percent. ${ }^{4}$ In some cases, however, (as shown in fig. 16) $\alpha$ increased considerably with applied r-f voltage. For these cases eq 1 does not hold. It is therefore advisable to make sure that $\alpha$ remains constant to at least 10 percent within the working voltage range required. This is in fact a requirement of matching the resistance, versus power functions of the thermistors under operating conditions and can be accomplished by comparing the value of $\alpha$ without $\mathrm{r}-\mathrm{f}$ voltage to that with maximum $\mathrm{r}-\mathrm{f}$ voltage at any low frequency, e. g., $100 \mathrm{kc}$ or lower. The potential error from this source increases with $V$.

\footnotetext{
${ }^{4}$ Equation $10^{7}$ on page 92 of the Technique of microwave measurements 11, Radiation Laboratory Series (McGraw-Hill Book Co., New York, N. Y., 1947), gives the thermistor resistance as a function of power dissipated in it. Computations made after the original manuscript was written indicate that this equation closely represents the characteristics of the thermistors in the mount described. The initial $\alpha$ of a pair of thermistors was computed and measured to be 1.04. It increased to about 1.1 at an $\mathrm{r}-\mathrm{f}$ voltage of $1.5 \mathrm{v}$. The potential error in this case was computed to be about $0.04 \%$.
} 
The required precision of bridge resistors must be of the same order of magnitude as that desired for $V$, because as shown in appendix IV,

$$
\frac{\Delta R_{T}}{R_{T}}=\frac{\Delta R_{b}}{R_{b}}=\frac{\Delta V}{V} \frac{\left(R_{T}+R_{b}\right)}{R_{b}},
$$

The factor $\left(R_{T}+R_{b}\right) / R_{b}$ is fixed by the operating thermistor characteristics and as used was around 1.7 .

\section{Factors Limiting High End of Voltage Range}

The top value of the standard $r-f$ voltage that can be determined with either the single- or the two-thermistor bridge is limited by the thermistor characteristics in a specific mount. With the smallest thermistors having dimensions mentioned above, this value was between 1.3 and $1.9 \mathrm{v}$, depending upon the temperature of components to which the bridge was connected. With thermistor beads having a diameter of about 116 in., voltages as high as $10 \mathrm{v}$ may be obtained. When proper capacity-type voltage dividers are employed, considerably higher voltages may be determined. These dividers will not be dealt with here.

\section{Factors Limiting Low End of Voltage Range}

The low limit of r-f voltages is of major importance. The lower it is, the more accurately voltages at the critically needed microvolt levels can be determined with the help of standard attenuators. Because of imperfections of standard attenuators, especially in the VHF and UHF regions, a considerably more accurate microvolt may be realized by using, for example, a standard millivolt and a $60-\mathrm{db}$ attenuator, rather than a standard volt and a $120-\mathrm{db}$ attenuator. This was the primary reason why considerable efforts were made to obtain as low a known voltage as possible. In doing so, another important objective was attained, namely, the opportunity of measuring the attenuation of standard attenuators directly in terms of voltage ratios. This is especially desirable where the permissible input power into an attenuator is limited.

The low end of the voltage range is limited by (a) the sensitivity of the thermistors and of the d-c bridge, (b) the ease with which low values of $V_{R 2}$ can be adjusted and the accuracy with which $V_{R 2}$ and $V_{R 1}$ can be measured, (c) stability and accuracy of $V_{0}$, (d) thermal voltages and incidental voltage drops in circuit leads and connections, and (e) ambient temperature stability.

\section{(a) Thermistor and Bridge Sensitivity}

The bridge sensitivity is proportional to the sensitivity of the thermistor arm to $r-f$ inputvoltage changes. For the single-thermistor bridge, this sensitivity (as indicated in the characteristic curves of fig. 16) is about $0.18 \mathrm{ohm} / \mathrm{mv}$ for operating values of thermistor resistances of from 50 to $100 \mathrm{ohms}$. For the matched two-thermistor bridge, the thermistor arm sensitivity is twice the single-thermistor sensitivity, i. e., $0.36 \mathrm{ohm} / \mathrm{mv}$, because the same $r-f$ voltage will cause an equal resistance increment in each thermistor.

The characteristics of the small-bead thermistors are such that an $R_{T}$ of $200 \mathrm{ohms}$ and $R_{b}$ of about 300 ohms were found most suitable for a twothermistor bridge arrangement. With the bridge and galvanometer used, changes in $R_{T}$ of the order of $0.01 \mathrm{ohm}$ in $200 \mathrm{ohms}$ could readily be detected. For the stated two-thermistor bridge sensitivity of $0.36 \mathrm{ohm} / \mathrm{mv}$, an $\mathrm{r}-\mathrm{f}$ voltage change of $14 \mathrm{mv}$ can be detected. The resulting uncertainty is therefore 0.1 percent for an applied voltage of $14 \mathrm{mv}$ and, of course, correspondingly less for higher applied voltages.

(b) Accuracy of $\mathrm{V}_{R 1}$ and $\mathrm{V}_{R 2}$

As already mentioned, a perfect voltage regulation of the source $V_{0}$ was assumed. A storage battery does approach this requirement, although any other monitored source could be applied if it meets stability requirements. One must keep in mind two difficulties when batteries are used. The first is the one stated before, namely, that $V_{0}$ cannot be conveniently adjusted except in approximate $2-\mathrm{v}$ steps. The second is the poor accuracy obtainable in trying to determine a small quantity when this small quantity is the difference between two measured relatively large quantities. This would be the case when both $V_{R 2}$ and $V_{R 1}$ were large. These two difficulties may be largely eliminated (as was done in this case) by the use of an unequal arm bridge. The initial bridge balance was obtained by varying $R_{b}$ with the adjustable resistor $\left(R_{1}, R_{2}\right)$ shorted. $V_{R 1}$ is therefore the voltage drop across the shorting switch. It can thereby be kept at a minimum and for relatively 


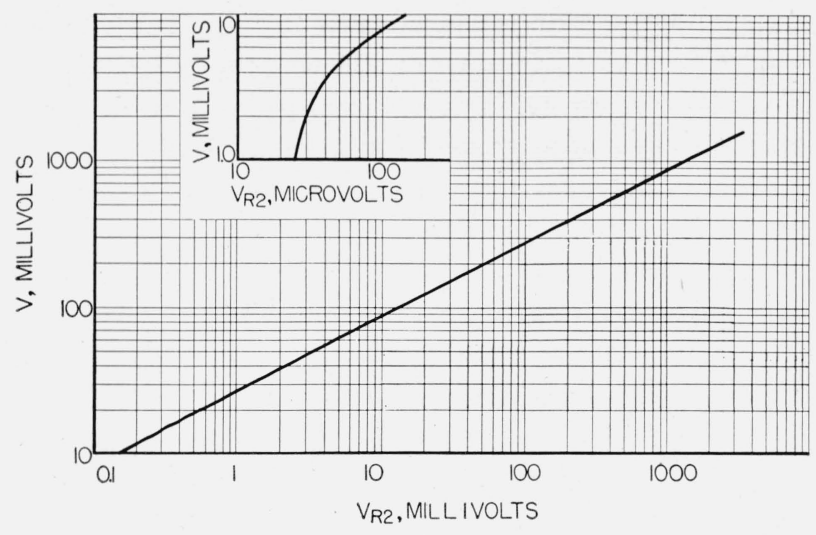

Figure 7. Values of $V_{R 2}$ corresponding to various values of $V$.

$V_{0}=10$ volts; $V_{R_{1}}=25$ microvolts; $R_{T}=200$ ohms; $R_{b}=300$ ohms; $\alpha=1.0$

$$
V=\frac{R_{T}}{\left(R_{T}+R_{b}\right)(1+\alpha)}\left(\alpha\left[V_{R 2}-V_{R_{1}}\right]\left[2 V_{0}-V_{R 2}-V_{R_{1}}\right]\right)^{\frac{1}{2}} .
$$

large measured voltages may be assumed equal to zero. As used, $V_{R 1}$ was of the order of $25 \mu \mathrm{v}$.

Figure 7 shows nominal d-c voltages $\left(V_{R 2}\right)$ that one must measure for the entire $r-f$ range at a given set of values of the bridge circuit. The curve is only nominal because $R_{b}$ is in practice somewhat different from the value chosen for this curve, depending on the characteristics of the particular thermistors in use. The case presented here is in many respects only typical. Modifications are possible in order to extend the low range; for example, a mount employing a large number of thermistors, all of them connected in series for direct current and in parallel for $\mathrm{r}-\mathrm{f}$ current, may be used, which would considerably increase the ratio of $R_{T}$ to the equivalent $\mathrm{r}-\mathrm{f}$ load resistance; lower values of $V$ may then be obtained for corresponding $V_{R^{2}}$ values. The greater part of the curve of figure 7 is linear on log-log paper. This may be expected from the interrelation (eq 2) when $V_{R 1} \cong 0$ and $2 V_{0}>>V_{R 2}$. Under these conditions the expression assumes the form

$$
V=K\left(2 V_{R 2} V_{0}\right)^{\frac{1}{2}}=K_{1}\left(V_{R 2}\right)^{\frac{1}{2}},
$$

or by

$$
\log V=\log K_{1}+\frac{1}{2} \log V_{R 2} .
$$

The degree of departure of $V$ from linearity below about $10 \mathrm{mv}$ is a function of the magnitude of $V_{R 1}$.
The effect of inaccuracy in measuring $V_{R 2}$ is shown in appendix $\mathrm{V}$. An error of 0.1 percent in $V$ is assumed, and the corresponding "permissible error" in determining $V_{R 2}$ is derived and plotted in figure 8 for values of $V$ from several millivolts to $1.5 \mathrm{v}$. The "potential error" is shown in figure 8 for values of $V$ from several millivolts to $1.5 \mathrm{v}$. The potential error shown in figure 8 is based on the performance of the particular potentiometer on hand with the present equipment. It was capable of measuring $V_{R 2}$ to $1 \mu \mathrm{v}$ or to 0.015 percent, whichever is greater. The intersection point of the two curves in figure 8 indicates that the minimum value of $V$ obtainable to 0.1 percent because of inaccuracy in measuring $V_{R 2}$ is approximately $20 \mathrm{mv}$. Lower levels of $V$ may no doubt be measured to the same accuracy with other potentiometers. The low limit may in that case be computed in a similar manner.

It is shown in appendix VI that, with the present equipment, a value of $V$ of $20 \mathrm{mv}$ can be measured to within 0.1 percent as a result of the inaccuracy in determining a voltage $V_{R_{1}}$ of the order of $25 \mu \mathrm{v}$.

Difficulties in adjusting $V_{R}$ will be discussed in the next section where the equipment components are described.

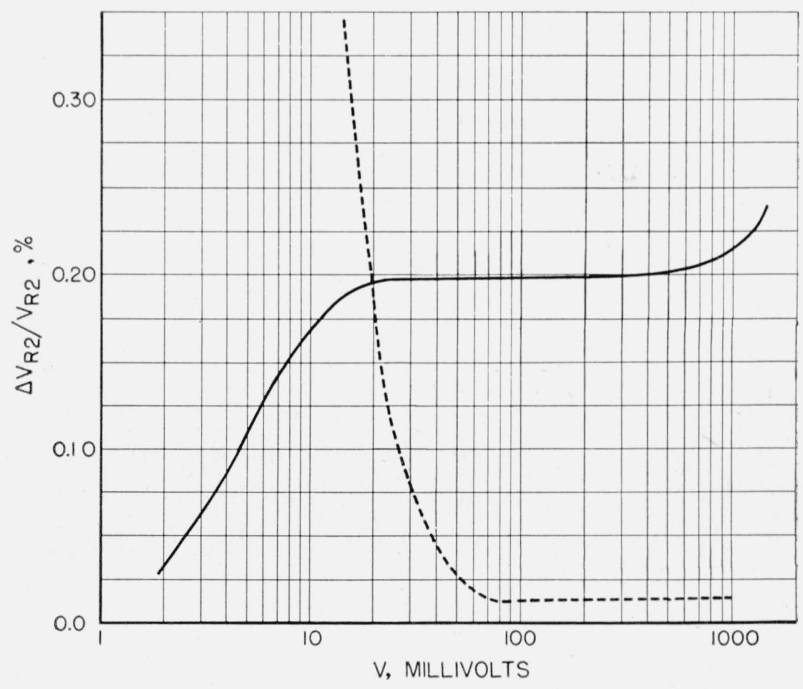

Figure 8. Required $V_{R 2}$ accuracy for $V$ accuracy of 0.1 percent.

Solid curve shows permissible error for $\Delta V / V$ equal 0.1 percent. Broken line shows potential error in measurement of $V_{R 2}$ as a result of limitations in d-c measuring equipment. $\quad V_{0}=10$ volts; $V R_{1}=25$ microvolts: $R_{T}=200 \mathrm{ohms}$; $R_{b}=300 \mathrm{ohms}$. 
(c) Accuracy and Stability of $V_{0}$

It can be shown that the accuracy with which $V_{0}$ must be measured for a desirable accuracy in $V$ is expressed by

$$
\frac{\Delta V_{0}}{V_{0}}=\frac{\Delta V}{V} \frac{V^{2}}{K V_{0}\left(V_{R_{2}}-V_{R_{1}}\right)}
$$

where $K=\left[R_{T} / 2\left(R_{T}+R_{b}\right)\right]$. For the present case of $\Delta V / V=10-{ }^{3}$ and $K=0.04$, the required accuracy of $V_{0}$ is 0.17 to 0.20 percent over the voltage range of about $1.5 \mathrm{v}$ to $20 \mathrm{mv}$, respectively. This accuracy is well within the limits of the equipment used.

The discharge rate of the storage batteries interferes with obtaining a bridge balance when the galvanometer circuit is adjusted for the high sensitivity required at low-voltage measurements.

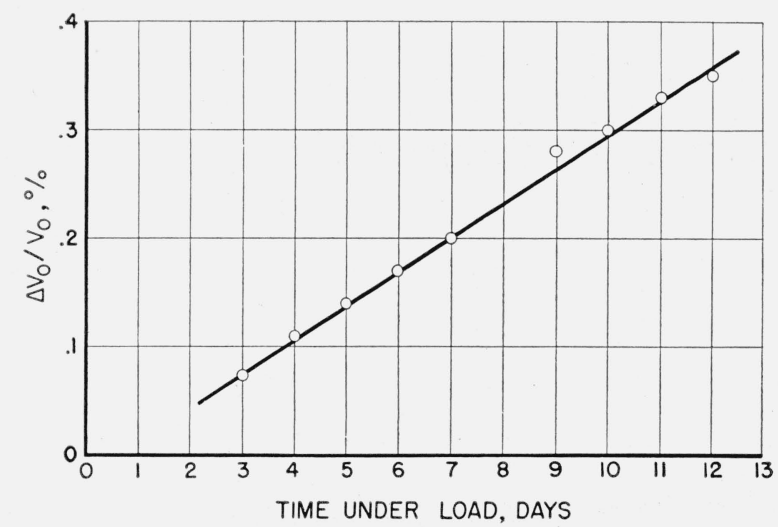

FIgure 9. Drop in terminal voltage with time of special batteries under a constant load of 45 milliamperes.

Figure 9 shows the drift of $V_{0}$ (under the present load) to be approximately 0.001 percent per hour. To evaluate this quantitatively one may assume that the drift is equivalent to a readjustment of $V_{R 2}$ required to restore bridge balance during a time interval of a single r-f voltage measurement. The application of a special $r-f$ and $d-c$ switch (described in the next section) reduces this interval essentially to the time required for a measurement of $V_{R 2}$. It may be seen from the curves given and the indicated drift that $V_{R 2}$ for a $V$ of $20 \mathrm{mv}$ will have to be determined in about $40 \mathrm{sec}$ if the drift is to be neglected. The steps leading to this value are as follows: The actual drift in $V_{0}=10 \mathrm{v}$ is $100 \mu \mathrm{v} / \mathrm{hr}$ (fig. 9). From figure 7 a voltage $V_{R 2}$ of about $550 \mu \mathrm{v}$ corresponds to a $V$ of $20 \mathrm{mv}$. The permissible error in this value of $V_{R 2}$ for a
0.1 percent error in $V$ is close to 0.2 percent or about $1.1 \mu \mathrm{v}$. The time allowed for a drift of not more than $1.1 \mu \mathrm{v}$ is therefore about $40 \mathrm{sec}$. Experimental observations indicate that common storage batteries have a drift of 5 to 10 times larger than the batteries used in our case. In addition they are considerably less stable. (The rate of voltage drop with time varied about 10 to 1 over a short time of observation.) It would thus be necessary to measure $V_{R 2}$ for a $V$ of $20 \mathrm{mv}$ in a fraction of a second. The importance of using low-discharge batteries of high stability and low internal resistance for very low voltage measurements is therefore apparent.

Summarizing the errors affecting the low limit of $V$, it is seen that a conservative estimate of the total possible error for all values down to $20 \mathrm{mv}$ caused by the determination of $\alpha, R_{T}, R_{b}, V_{R 1}$, $V_{R 2}, V_{0}$, and by limited bridge sensitivity and instability of $V_{0}$ will not exceed 0.8 percent assuming the individual 0.1 percent errors to be all positive or all negative. It is shown in section IV that errors contributed by incidental thermal voltages, connecting-lead voltage-drops, and ambient temperature variations of $\pm 1^{\circ} \mathrm{F}$. are negligible in comparison with others. An extension of $V$ below $20 \mathrm{mv}$ without sacrificing accuracy will require first further improvement of $V_{0}$ stability and, second, the improvement of the accuracy of measuring $V_{R 1}$ and $V_{R 2}$.

\section{Measuring Equipment and Results}

\section{Description of Equipment}

The complete arrangement of the two-thermistor bridge is shown in figures 10 and 11 . Figure 10 is a view of the experimental setup including a vacuum-tube voltmeter under calibration. A final working arrangement could no doubt be consolidated to a great extent. Figure 11 is a combination schematic and block diagram in which the designations of the components correspond with those of figure 10 . The source of $r-f$ voltage consisting of a generator, filter, and matching networks is not shown in figure 10 .

The major components of the equipment were:

(a) Resistance boxes comprising the legs $R_{T}$ and $R_{b}$ of the bridge.

(b) Resistance boxes and a helical type singlewire adjustable resistance comprising the resistance $R$ in series with the battery 


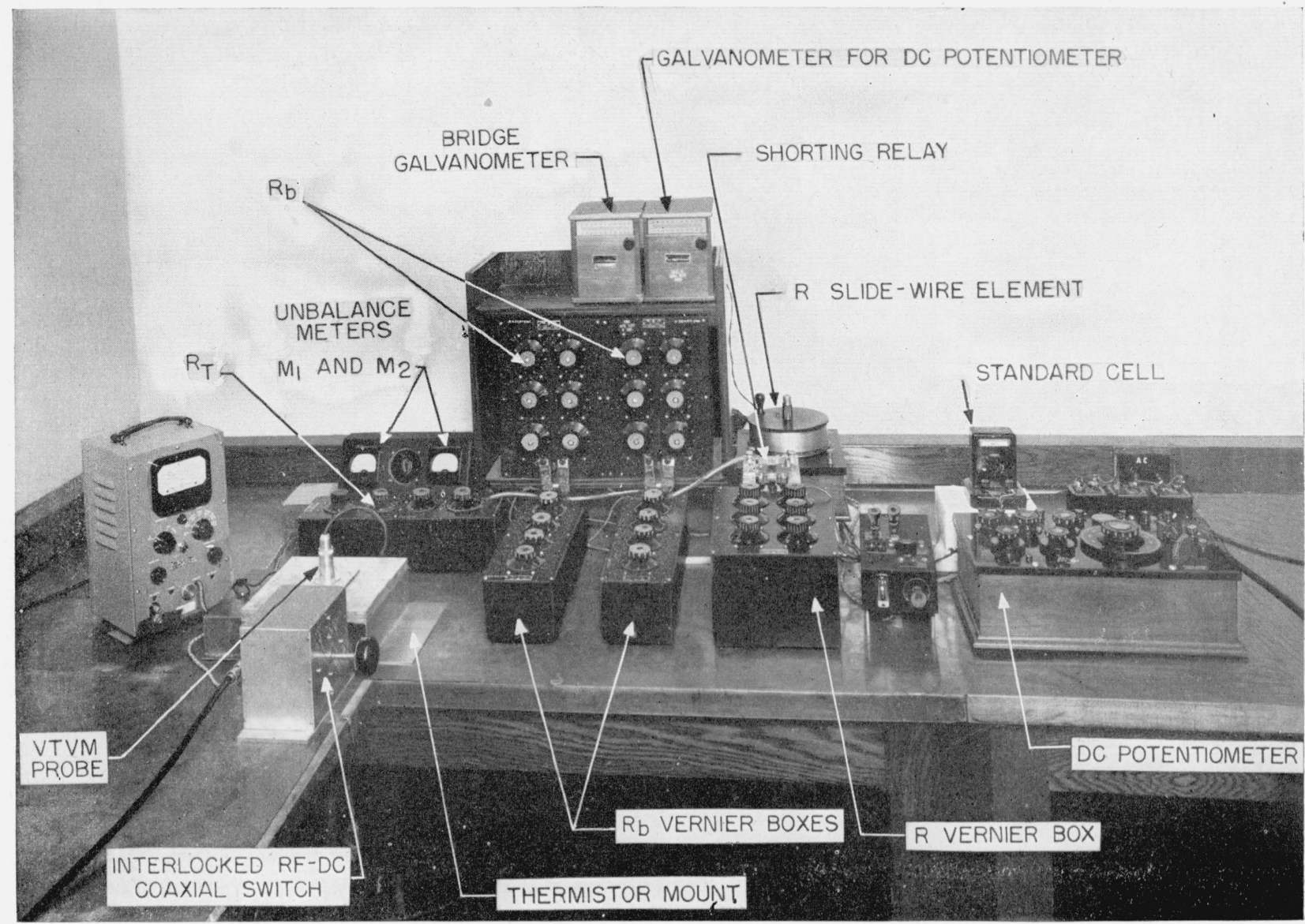

FIGURE 10. Radio-frequency voltage thermistor-bridge equipment.

$V_{0}$ used to vary the voltage applied to the bridge. Voltage drops $V_{R 1}$ and $V_{R 2}$ (used in eq 1, 2, 3, and 4) are measured across this resistance.

(c) D-c potentiometer, associated resistors, $R_{p 1}$ and $R_{p 2}$ to increase its voltage range, a plug box used for conveniently connecting the potentiometer for measurement of $V_{0}, V_{R 1}, V_{R 2}$, and of $\left(V_{0}-V_{R}\right)$ when desirable, and a standard cell.

(d) An interlocked, r-f, d-c switch used to replace instantaneously the r-f power fed to the bridge by its equivalent $d$-c power or vice versa without upsetting the bridge balance.

(e) A shorting relay controlled by the interlocked r-f, d-c switch, mentioned in item (d); this relay shorts the resistor $R$ in series with the bridge battery, thereby increasing the voltage applied to the bridge while the $r-f$ power is removed from the bridge.

(f) The thermistor mount.

(g) High-resistance d-c voltmeters to indicate the ratio of $R_{T 1}$ to $R_{T 2}$.

(h) Switch $\mathrm{S}_{0}$ used to connect the battery $V_{0}$ to the bridge.

(i) Switch $\mathrm{S}_{D}$ used to connect a standby load $R_{D}$ to the battery $V_{0}$ in place of the bridge. This load was necessary to prevent a slight increase in battery voltage when standing idle for several hours. On connecting a load to an idle battery the rate at which the terminal voltage decreases is relatively high during the initial discharging period.

The operational procedure was briefly as follows. Referring to figures 10 and 11 , with no voltage applied to the bridge, $R_{T}$ and both $R_{b}$ arms were set to predetermined values that would approx- 


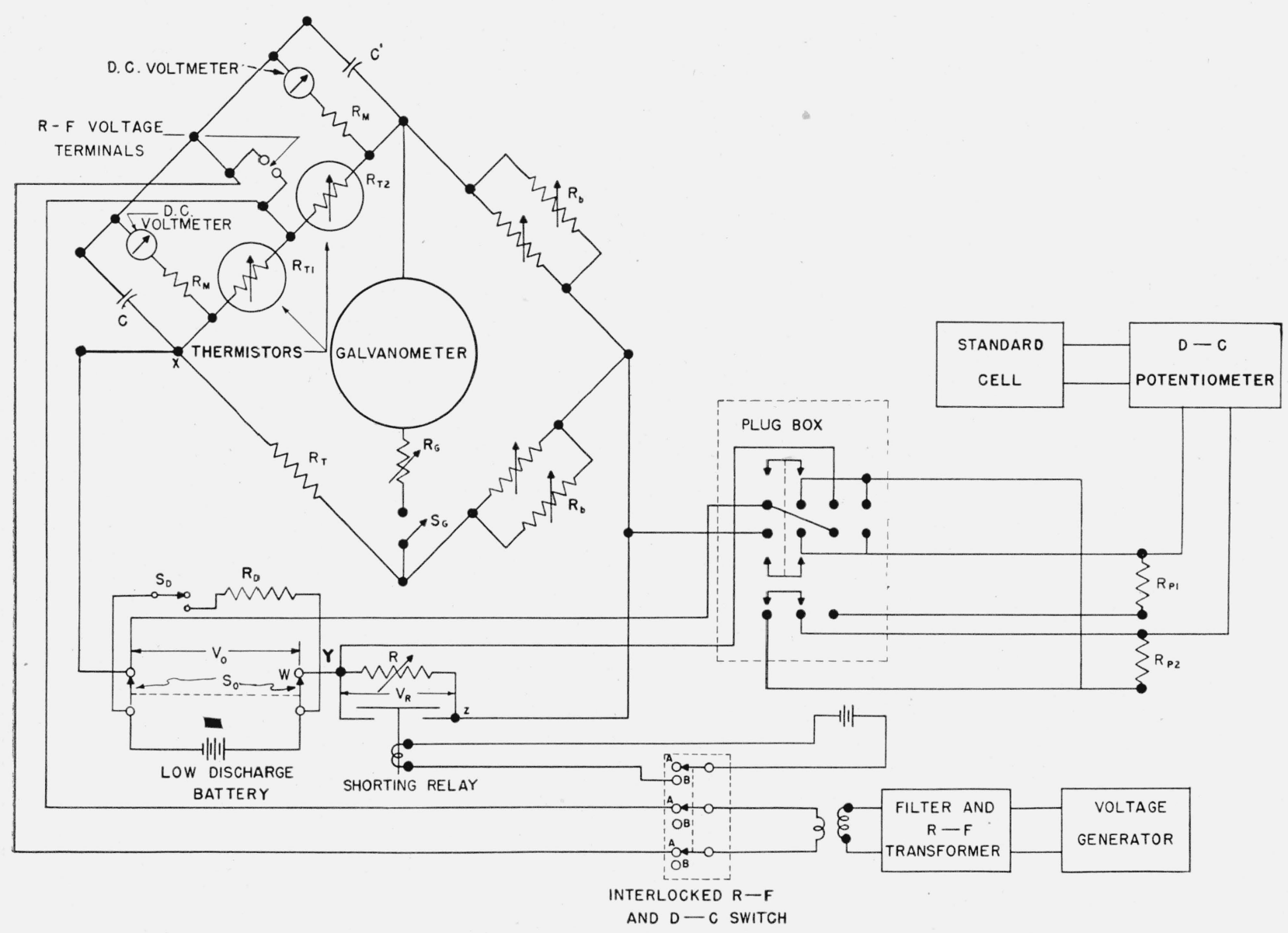

Figure 11. Operational schematic and block diagram of the r-f voltage thermistor-bridge equipment.

$C, C^{\prime}=$ mica 0.01 microfarad condensers; $R_{T}, R_{b}=$ bridge ratio arms; $R=$ series dropping resistors; $R_{P 1}, R_{P 2}=$ d-c voltage divider used with d-c potentiometer; $R_{G}=$ galvanometer sensitivity control; $S_{G}=$ galvanometer switch.

imately balance the bridge at the specific d-c voltage to be applied. With the shorting relay closed, the interlocked r-f, d-c switch in position $B$ (fig. 11), and the sensitivity of the galvanometer reduced, switch $S_{0}$ was closed. Both $R_{b}$ arms were adjusted simultaneously until a rough balance was obtained. After increasing galvanometer sensitivity by reducing $R_{g}$, further adjustments in $R_{b}$ were made until a satisfactory balance resulted. R-f was then applied in small increments after closing the coaxial switch and equilibrium restored by increasing the value of $R$. Several operations of the interlock switch were necessary until a desirable balance was observed. In calibrating a vacuum-tube voltmeter, the amount of $r-f$ applied depended upon the meter deflection desired. The amount of $\mathrm{d}$-c voltage removed from the bridge to restore balance was then measured by the potentiometer.
Cross sections of the thermistor mount are shown figures 12 and 13 . The plate, $A$, and the pin, $L$ (fig. 12) are removable, as is illustrated in figure 14, to permit means of connecting various probes of voltmeters under calibration to the mount. The line drawing in the lower corner of figure 14 is an enlargement of the gap in which the thermistors

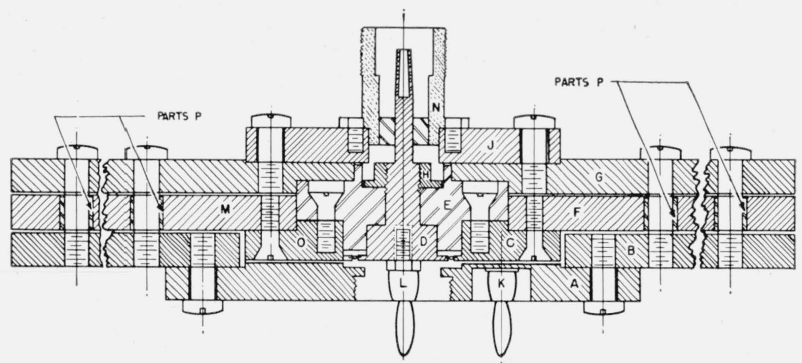

Figure 12. Cross-sectional drawing showing the construction of the thermistor mount. 


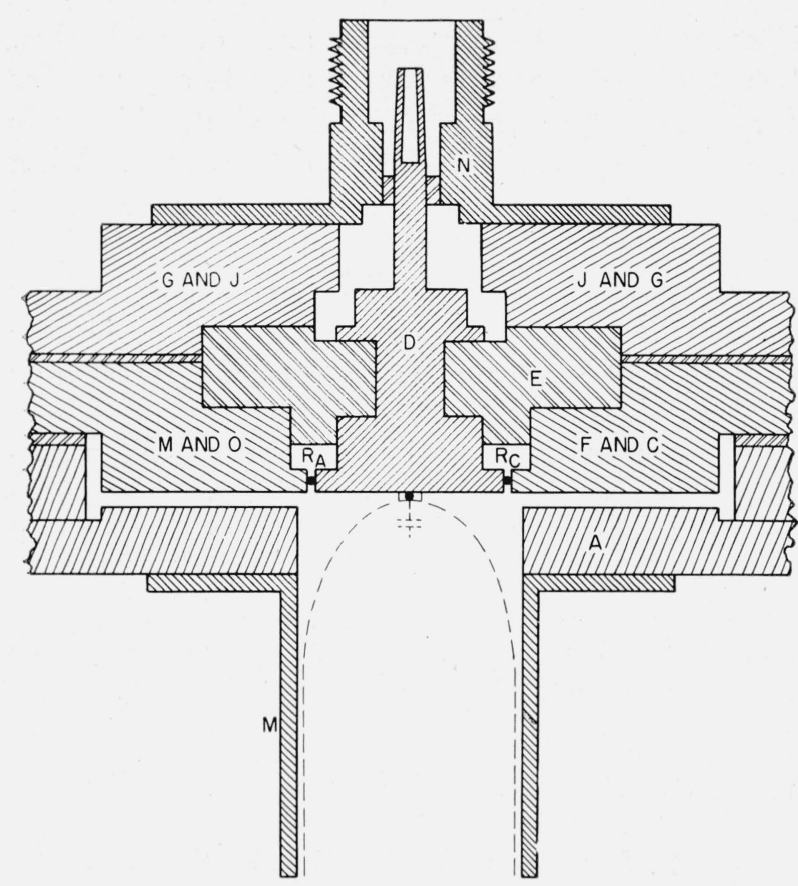

Figure 13. Cross-sectional drawing of the core of the mount showing the location of the thermistors and their proximity to the point where external connections are made for calibration purposes.

A dashed outline is shown of a typical VTVM probe in position for calibration. R-f power is fed into fitting $N$.

are mounted, whereas the part in the upper right corner (fig. 14) shows the other side of the core assembly. The remainder of the mount consists

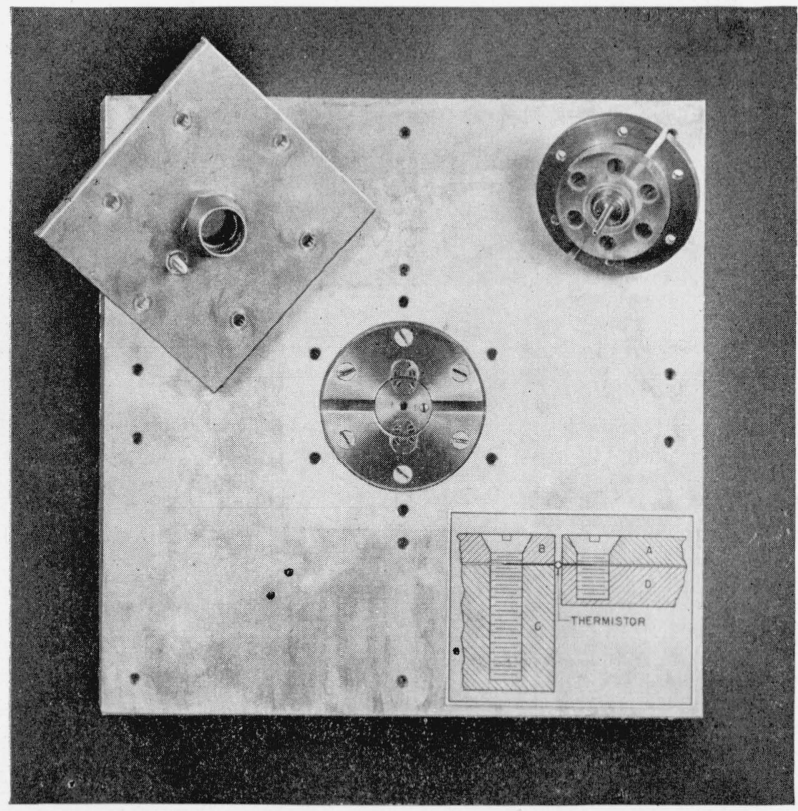

FIGURE 14. Thermistor mount partly disassembled. of an assembly of the mica blocking condensers $C$ and $C^{\prime}$ shown in figures 5 and 11 . One thermistor is connected to parts $D$ and $O-M$ (fig. 12); the other to $D$ and $C-F$. Parts $B, F$, and $G$ and $B, M$, and $G$, figure 12 , comprise the condensers $C$ and $C^{\prime}$ of about $0.01 \mu \mathrm{f}$ each. At low frequencies where the reactance of $C$ and $C^{\prime}$ becomes appreciable, additional external capactance was added in parallel with them.

The r-f admittance of the thermistor bridge was measured in the VHF range employing a slotted coaxial transmission line. The input conductance was found to equal the d-c value of the two thermistors in parallel within the limits of accuracy of the measuring equipment.

Figure 15 illustrates the effect the large metal mass of the mount had on the thermistor characteristics. The broken line curve is a typical

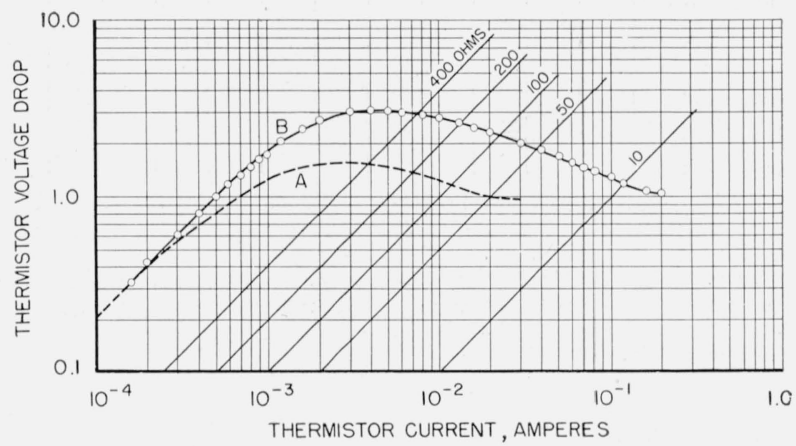

Figure 15. Thermistor characteristic curves.

A, thermistor unmounted (manufacturers data); B. thermistor mounted close to large heat-conducting surfaces of mount (fig. 14).

characteristic of the thermistor, unmounted, at $25^{\circ} \mathrm{C}$. whereas the solid curve is that obtained with the thermistors in the mount. The difference was due to the large heat dissipation of the metal plates to which the thermistors were connected. The decrease in sensitivity that resulted was more than compensated for by a saving of about 80 percent of the time required to obtain thermal equilibrium of the thermistors during initial balance adjustments.

It was found experimentally that $\alpha$ increased as the $\mathrm{r}$-f voltage level was increased $(\alpha \geqslant 1)$. Figure 16 shows $R_{T 1}$ and $R_{T 2}$ at different values of $V$ for an appreciably mismatched pair of thermistors. This effect was found to be independent of frequency. As was previously explained, individual pairs of thermistors were chosen having characteristics sufficiently similar to eliminate large 
values of $\alpha$ requiring high-precision d-c voltmeters, as well as large ratios of $\alpha_{2} / \alpha_{1}$, where $\alpha_{1}$ is the ratio of the two thermistor resistances without $r-f$ applied to the bridge, and $\alpha_{2}$ is the ratio with $\mathrm{r}-\mathrm{f}$ applied. For $\alpha_{2} / \alpha_{1}$ of 2.0 , an error in $V$ of 4 percent resulted. Two precautions were necessary in connection with the use of these voltmeters for measuring $\alpha$. The first was that the $\mathrm{d}-\mathrm{c}$ resistance of these instruments be large compared with $R_{T 1}$ and $R_{T 2}$ so as not to introduce an appreciable error in the thermistor resistance values. The second was that there be a constant low-resistance d-c path through the $r-f$ source if the d-c instruments were to measure the drop across $R_{T 1}$ and $R_{T 2}$.

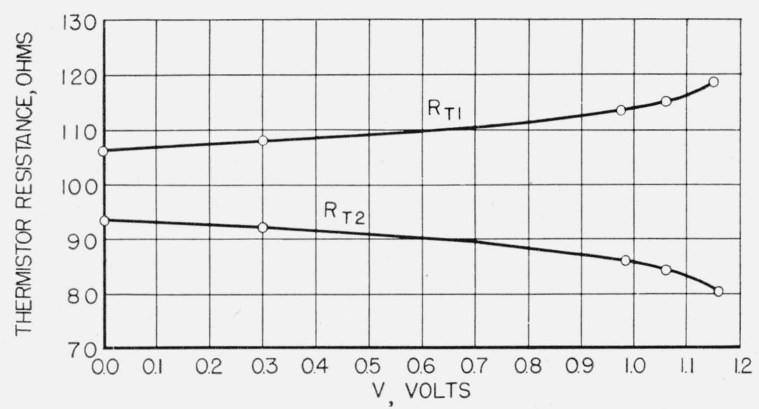

Frgure 16. Resistance of individual thermistors, connected in parallel, versus $V$.

$R_{T 1}+R_{T 2}=R_{T}=200$ ohms (fig. 11).

The voltage $V_{R 2}$ was controlled by varying the resistance $R$ (fig. 11) in series with the bridge (fig. 10). The greatest difficulty occurred at low values of $V$, where the resistance $R$ was of the order of $1 \mathrm{ohm}$ or less. As shown in figure 11, a low-resistance slide-wire in parallel with a resistance box of high value was used, the box serving as a vernier on the slide-wire.

The shorting relay (fig. 11) is a special relay with mirror-smooth silver-plated contact surfaces. Such construction was necessary to insure a low, constant voltage drop when the relay was closed; otherwise measurement of $V_{R 1}$ would have been necessary each time the relay was operated. The value of $V_{R 1}$ for typical, bridge-operating conditions $\left(R_{T}=200 \mathrm{ohms} ; R_{b}=300 \mathrm{ohms}\right.$ ) was found to be about $25 \mu v$ and remained stable to within the measuring accuracy of the potentiometer used.

The voltages $V_{0}, V_{R 2}$, and $V_{R 1}$ were measured by the d-c potentiometer, $P$, in terms of the standard cell (fig. 11) at points $x, y$, and $z$. To reduce the resistance of leads, wherever advisable, wide low-resistance straps were employed for interconnecting the resistance arms of the bridge. The average resistance contributed to any arm by the leads was no more than $0.005 \mathrm{ohm}$, which was negligible compared with 200 to $300 \mathrm{ohms}$. With the present setup, it was found that the internal resistance of the battery and the resistance of the leads used to connect the battery to the bridge totaled less than $0.05 \mathrm{ohm}$ and justified the assumption of negligible voltage regulation for the loads used.

The equipment was installed in an air-conditioned room with the temperature controlled to within $1 \mathrm{deg}$ C. This precaution served the dual purpose of, first, eliminating the effect of ambient temperature variations on the thermistors, and, second, reducing variations of $V_{0}$ and thermal voltages within the circuit. To reduce further the effect of thermal emf's, every effort was made to remove contacts of dissimilar metals.

\section{Agreement With Other Methods}

Voltage measurements by independent methods, including the use of a single-thermistor bridge, were used as a check on the two-thermistor bridge. The results obtained are presented in table 1.

The principles involved in methods 1 and 2 of table 1 are indicated by their titles in the table and have been described in previous literature (see footnote 1). The agreement between the two-thermistor and the single-thermistor bridges

\section{TABLE 1.}

\begin{tabular}{|c|c|c|c|}
\hline Method & 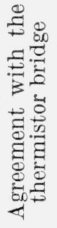 & $\begin{array}{c}\text { Frequency range } \\
\text { of agreement }\end{array}$ & Range-extension devices \\
\hline $\begin{array}{l}\text { Deflection of cathode- } \\
\text { ray beam. }\end{array}$ & $\begin{array}{r}\text { Per- } \\
\text { cent } \\
1\end{array}$ & $100 \mathrm{ke}$ to $200 \mathrm{Mc}$ & $\begin{array}{l}\text { Capacity-type voltage } \\
\text { dividers of about } \\
10: 1 \text {, and r-f trans- } \\
\text { formers. }\end{array}$ \\
\hline $\begin{array}{l}\text { Measurement of cur- } \\
\text { rent through a } \\
\text { known resistance. }\end{array}$ & 1 & $100 \mathrm{kc}$ to $30 \mathrm{Mc}$. & None. \\
\hline $\begin{array}{l}\text { Transmission-line } \\
\text { voltage-distribu- } \\
\text { tion. }\end{array}$ & 1 & $100 \mathrm{Me}$ to $600 \mathrm{Mc}$ & Do. \\
\hline $\begin{array}{l}\text { Single-thermistor } \\
\text { bridge. }\end{array}$ & 1 & $100 \mathrm{ke}$ to $30 \mathrm{Me}$ & Do. \\
\hline
\end{tabular}


was verified to $30 \mathrm{Mc}$. The difficulties encountered with the single-thermistor bridge for frequencies above $30 \mathrm{Mc}$ were previously pointed out in section III.

(a) Transmission-Line-Voltage-Distribution Method

The third listed independent method is based upon the interrelation between the $\mathrm{r}-\mathrm{f}$ power propagated along a transmission line (having a known characteristic impedance and negligible attenuation) and the voltage distribution along this line. A slotted transmission line is used, and the voltage ratios at any two given points are accurately determined by moving a probe along the slot. The conventional way to determine the ratios is either by using a standard attenuator or a calibrated probe-output detector. A more direct, and therefore a more accurate, way is to determine these voltage ratios from measurements of line distances translated in turn by computation into equivalent electrical lengths. Thus absolute values of voltages at a given point along the line may be computed from: (a) the characteristic impedance of the line, (b) power, determined by d-c measurements, and (c) distances measured along the line. The interfering factor in this otherwise potentially highly accurate method is the nonavailability of slotted transmission lines with sufficiently uniformly distributed constants. The frequency range is limited at the low end by the physical length of the line.

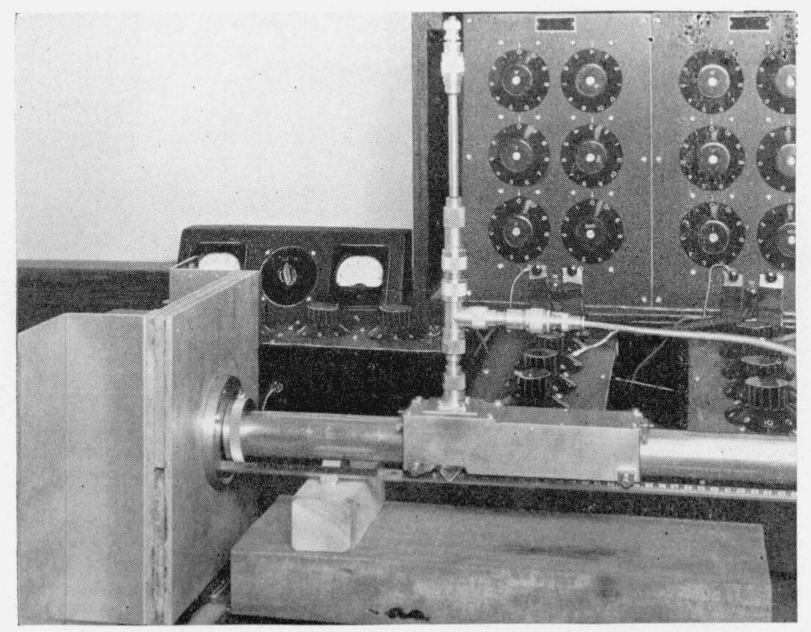

Figure 17. Thermistor mount connected to a slotted transmission line for measurement of agreement between two independent methods of voltage measurements and for standard impedance measurements.
For purposes of comparison with the bridge measurements, the thermistor mount was connected to a slotted transmission line as illustrated in figure 17. A line extension designed for use with the line was so connected to the mount that the uniform line was carried to within $0.003 \mathrm{in}$. of the core of the mount. Therefore the voltage at the end of the line was essentially the same as that at the thermistors.

The measurement procedure was as follows:

(1) The thermistor mount, having been internally shorted where the thermistors connect, was placed directly at the end of the slotted transmission line, and the position $\lambda / 2$ from the load determined (step $A$, fig. 18).

(2) With the short removed, the thermistor bridge was used as an r-f load and power meter. The voltage standing-wave ratio $\left(V_{\max } / V_{\min }=\rho\right)$, the voltage ratio $\left(V_{A} / V_{\max }=K_{1}\right)$, and the power delivered to the load, were measured (steps $B$ and $C$, fig. 18).

The magnitude of the standard voltage, $V$, was calculated from eq 9. (See derivation in appendix VII).

$$
V=K_{1}\left(Z_{0} P_{R F} \rho\right)^{\frac{1}{2}},
$$

where $K_{t}=V_{A} / V_{\max }$, the ratio of the voltage $\lambda / 2$ from the load to the maximum voltage in the line. $Z_{0}=$ characteristic impedance of the line.

$\rho=$ ratio of maximum to minimum voltage in the line.

$P_{R F}=$ r-f power transmitted to the load $=$ bridge d-c power decrement.

The agreement between the two methods up to $600 \mathrm{Mc}$ was 1 percent or better, whereas up to $700 \mathrm{Mc}$ it was better than 2 percent (fig. 19).

The transmission-line voltage-distribution method was the only one used to verify the accuracy of the bridge at frequencies above $200 \mathrm{Mc}$. Because the transmission line used was known to have an insufficiently uniform distribution of constants, the bridge measurements were considered the more reliable of the two. One may apply the transmission-line method for direct calibration of voltmeters with some sacrifice in accuracy using an arrangement similar to that illustrated in figure 20. The probe of the voltmeter under calibration is connected at the end of the slotted line in place of the thermistor mount. In parallel with this probe and physically as close as possible to it, a cable is connected to feed the r-f power 

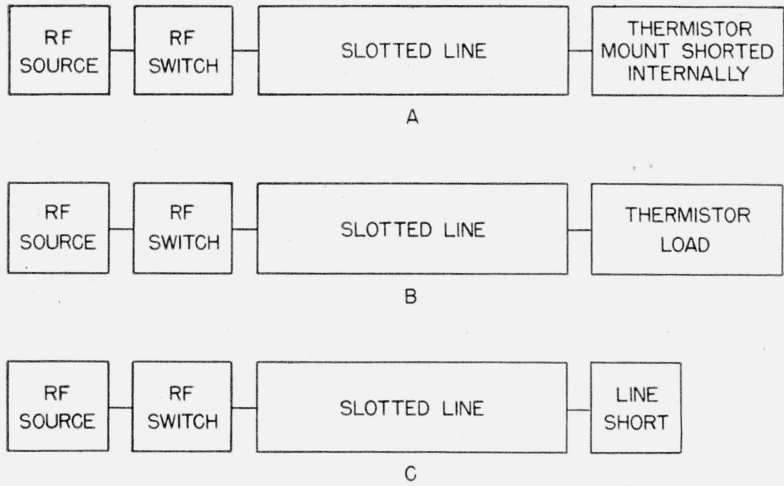

FIgURE 18. Block diagram of equipment and steps to ascertain agreement between bridge method and voltagedistribution-in-a-slotted-line method of voltage measurements.

from the line to any available accurate r-f powermeasuring device. In figure 20 the thermistor bridge is shown as such a device. The procedure and computations may be the same as described above. One must correct for possible power losses in the voltmeter probe if these are appreciable.

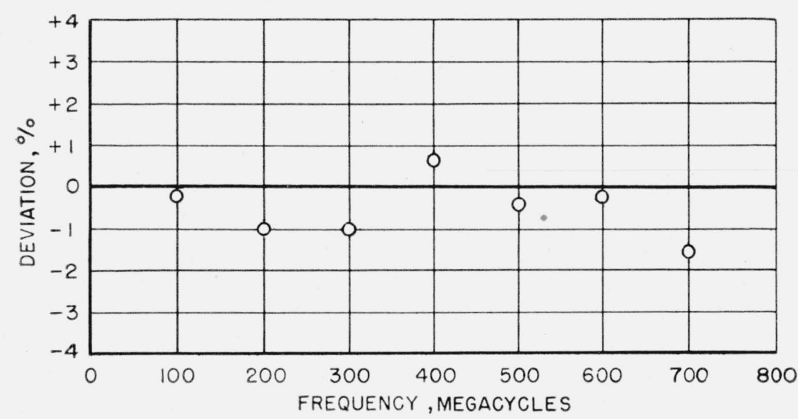

Figure 19. Deviation in percentage of $V_{p}$ from $V_{T}$.

$V_{p}=V$ obtained from power and voltage-distribution measurement; $V_{T}=V$ obtained with bridge measurement.

\section{Standardization of a V-T Voltmeter}

A calibration curve of a V-T voltmeter obtained with the bridge from $100 \mathrm{kc}$ to $800 \mathrm{Mc}$ is shown in figure 21 . The applied standard voltage required to obtain a fixed deflection of the voltmeter had to be increased in the frequency range of about 50 to $400 \mathrm{Mc}$ as a result of the transit-time effect in the VTVM probe diode. At higher frequencies the standard voltage had to be decreased because of the natural resonance of the diode and input terminals of the probe.

In conclusion, we feel confident that standard voltages could be obtained with this two-thermis- tor bridge at frequencies considerably above 800 Mc with accuracies equal to those at the lower frequencies.

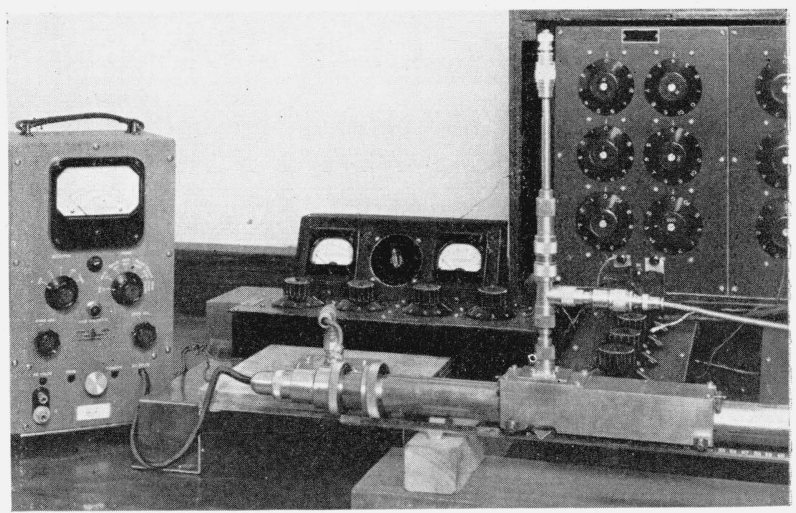

FIGURE 20. VTVM-probe connected to the slotted transmission line for calibration using power and voltagedistribution technique.

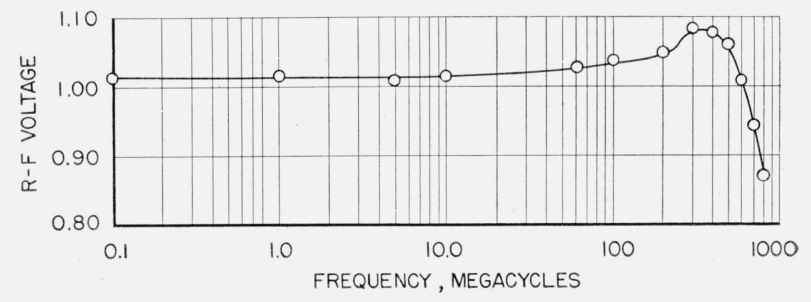

FIGURE 21. Calibration curve of a vacuum-tube voltmeter obtained with the bridge.

\section{Appendix I. General Expression for Bo- lometer Bridge r-f Voltage}

$$
W_{1}=\frac{V_{b 1}^{2} R_{T}}{\left(R_{T}+R_{b}\right)^{2}}
$$

$=\mathrm{d}$-c power dissipated in the thermistors with no $\mathrm{r}$-f applied. (See fig, 5 and definition of terms below eq 1 of text).

$$
W_{2}=\frac{V_{b 2}^{2} R_{T}}{\left(R_{T}+R_{b}\right)^{2}}
$$

$=\mathrm{d}$-c power dissipated in the thermistors with $\mathrm{r}-\mathrm{f}$ applied, where $V_{b 2}=V_{0}-V_{R 2}$ and $V_{b 1}=V_{0}-V_{R 1}$

Substituting for $V_{b 2}$ and $V_{b 1}$ in eq 10 and 11.

$$
\begin{gathered}
\begin{aligned}
& W_{1}= \frac{R_{T}}{\left(R_{T}+R_{b}\right)^{2}}\left(V_{0}-V_{R 1}\right)^{2} \\
& W_{2}= \frac{R_{T}}{\left(R_{T}+R_{b}\right)^{2}}\left(V_{0}-V_{R 2}\right)^{2} . \\
& \text { If } W=W_{1}-W_{2}, \\
& \text { and } W=\frac{V^{2}}{R_{L}} \text { where } \frac{1}{R_{L}}=\frac{R_{T}}{R_{T 1} R_{T 2}},
\end{aligned}
\end{gathered}
$$


substituting for $W_{1}$ and $W_{2}$ in eq 14 and solving for $V$ yields:

$$
V=\frac{1}{R_{T}+R_{b}} \sqrt{R_{T} R_{L}\left(V_{R 2}-V_{R 1}\right)\left(2 V_{0}-V_{R 2}-V_{R 1}\right)} .
$$

Let $R_{T 1}=\alpha R_{T 2}$, where $\alpha \geq 1$ and remains constant for all values of applied $r-f$ voltage. It can be shown that eq 14 holds in this case.

Then $R_{L}=[\alpha /(1+\alpha)] R_{T 2}$ or $R_{L}=\left[\alpha /(1+\alpha)^{2}\right] R_{T}$

Substituting in equation eq 16

$$
V=\frac{R_{T}}{\left(R_{T}+R_{b}\right)(1+\alpha)} \sqrt{\alpha\left(V_{R 2}-V_{R 1}\right)\left(2 V_{0}-V_{R 2}-V_{R 1}\right)} .
$$

\section{Appendix II. Error in $V$ When $\alpha=R_{T 1} / R_{T 2}$ Is Neglected}

In eq 1 let

$$
\frac{R_{T}}{\left(R_{T}+R_{b}\right)}\left[\left(V_{R 2}-V_{R 1}\right)\left(2 V_{0}-V_{R 2}-V_{R 1}\right)\right]^{\frac{1}{2}}=K,
$$

then

$$
V=K \alpha^{\frac{1}{2}}(1+\alpha)^{-1} .
$$

Let also $V$ for $\alpha=1$ be designated as $(V)_{1}$

then

$$
(V)_{1}=\frac{1}{2} K
$$$$
\text { and }
$$

$$
\frac{\Delta V}{V}=\frac{(V)_{1}-V}{(V)_{1}}=\frac{\frac{1}{2} K-K \alpha^{\frac{1}{2}}(1+\alpha)^{-1}}{\frac{1}{2} K}=1-2 \alpha^{\frac{1}{2}}(1+\alpha)^{-1} .
$$

Substituting values of $\alpha$ from 1 to 2 , the error in percent of $V$ is computed and is shown in figure 6 .

\section{Appendix III. Error in Measured $V$ as a Result of Inaccuracy in Measuring $\alpha$}

From eq 18, appendix II,

$$
\frac{\Delta V}{V}=\Delta \alpha\left[\frac{1-\alpha}{2 \alpha(1+\alpha)}\right]
$$

which holds for $\Delta \alpha \leqq 0.02$

Thus

$$
\frac{\Delta \alpha}{\alpha}=\frac{\Delta V}{V} \frac{2(1+\alpha)}{1-\alpha}
$$

and for an error of 0.1 percent in $V$ caused by an inaccuracy in measuring $\alpha$ this becomes

$$
\frac{\Delta \alpha}{\alpha}=0.001\left[\frac{2(1+\alpha)}{(1-\alpha)}\right],
$$

which is plotted in figure 6 versus $\alpha$.

For larger values of $\alpha$, the dfferential approach is incorrect. From eq 18, appendix II

$$
V=K \alpha^{\frac{1}{2}}(1+\alpha)^{-1} \text {. }
$$

As $\alpha$ increases, $V$ decreases. $\frac{V}{V_{1}}>1$, where $V_{1}=$ the cal- culated value of the $\mathrm{r}-\mathrm{f}$ voltage when $\alpha>1$ for the condition where $\Delta V / V=10^{-3}$.

$$
\frac{V}{V_{1}}-1=10^{-3}
$$

Substituting for $V$ and $V_{1}$ in terms of $\alpha$

$$
\frac{\alpha^{\frac{1}{2}}(1+\alpha)^{-1}}{(\alpha+\Delta \alpha)^{\frac{1}{2}}(1+\alpha+\Delta \alpha)^{-1}}-1=10^{-3} .
$$

Reducing to a common denominator and substituting

$$
\left(\frac{1}{\alpha}+1\right)=\sigma \text { and } \frac{\Delta \alpha}{\alpha}=y .
$$

Then $\frac{(\sigma+y)^{2}}{\sigma^{2}(1+y)}=1.002$.

Solving for $y$ in terms of $\sigma$

$$
y=\frac{-\left(2 \sigma-1.002 \sigma^{2}\right) \pm \sqrt{\left(2 \sigma-1.002 \sigma^{2}\right)^{2}+0.008 \sigma^{2}}}{2}
$$

\section{Appendix IV. Accuracy of $V$ as a func- tion of accuracy of $R_{T}$ and $R_{b}$}

In eq 1 let the factor in the square bracket $=K$ and $\alpha=1$, then

and

$$
V=\frac{1}{2} K R_{T}\left(R_{T}+R_{b}\right)^{-1},
$$

$$
\Delta V=\Delta R_{T}=\frac{1}{2} K\left[\left(R_{T}+R_{b}\right)^{-1}-R_{T}\left(R_{T}+R_{b}\right)^{-2}\right] .
$$

Therefore

$$
\frac{\Delta R_{T}}{R_{T}}=\frac{\Delta V}{V} \frac{R_{T}+R_{b}}{R_{b}} .
$$

Similarly

$$
\frac{\Delta R_{b}}{R_{b}}=-\frac{\Delta V}{V} \frac{R_{T}+R_{b}}{R_{b}} .
$$

For the apparatus presently in use, $\left(R_{T}+R_{b}\right) / R_{b}$ is approximately $5 / 3, R_{T}=200 \mathrm{ohms}$ and $R_{b}=300 \mathrm{ohms}$.

Dropping the minus sign as of no significance (errors will be added),

$$
\frac{\Delta R_{T}}{R_{T}}=\frac{\Delta R_{b}}{R_{b}}=1.7 \frac{\Delta V}{V} .
$$

Appendix V. Derivation of $\Delta V_{R 2} / V_{R 2}$ in terms of $V$

From eq 1

where

$$
V^{2}=K\left(2 V_{0}-V_{R 2}-V_{R 1}\right)\left(V_{R 2}-V_{R 1}\right),
$$

$$
\begin{gathered}
K=\left[\frac{R_{T}}{2\left(R_{T}+R_{b}\right)}\right]^{2} \text { when } \alpha=1, \\
\therefore V \delta V=K\left(\bar{V}_{0}-V_{R 2}\right) \delta V_{R 2} . \\
\Delta V_{R 2}=\Delta V \frac{\delta V_{R 2}}{\delta V}=\Delta V^{r} \frac{V}{K\left(V_{0}-V_{R 2}\right)} \\
\frac{\Delta V_{R 2}}{V_{R 2}}=\frac{\Delta V}{V} \frac{V^{2}}{K V_{R 2}\left(V_{0}-V_{R 2}\right)} .
\end{gathered}
$$

Figure 8 (solid line) is a plot of this equation for

$$
\frac{\Delta V}{V}=0.1 \%
$$




\section{Appendix VI. Error in $V$ Caused by Inaccuracy of Measuring $V_{R 1}$}

From eq 33

$$
\begin{gathered}
\Delta V_{R 1}=\Delta V \frac{\partial V_{R 1}}{\partial V}=\Delta V \frac{V}{K\left(V_{R 1}-V_{0}\right)}, \\
\therefore \frac{\Delta V_{R 1}}{V_{R 1}}=\frac{\Delta V}{V} \frac{V^{2}}{K V_{R 1}\left(V_{R 1}-V_{0}\right)} .
\end{gathered}
$$

In the case under consideration $V_{0} \gg V_{R 1}$, because $V_{0}=10$ $\mathrm{v}$ and $V_{R 1}=25 \times 10^{-6} \mathrm{v}$. $V_{R 1}$ can be measured to $1 \mu \mathrm{v}$ or $\Delta V_{R 1} / V_{R 1}= \pm 1 / 25$

$$
\therefore \frac{\Delta V_{R 1}}{V_{R 1}} \doteq-\frac{\Delta V}{V} \frac{V^{2}}{10 K V_{R 1}} .
$$

Neglecting the minus sign because the error can be positive or negative and assuming $\Delta V / V=10^{-3}$ we have

$$
\begin{aligned}
\frac{1}{25} & =10^{-3} \frac{V^{2}}{10 K \times 25 \times 10^{-6}}, \\
K & =0.04 \\
\therefore V & =2 \times 10^{-2} \mathrm{v}=20 \mathrm{mv}
\end{aligned}
$$

\section{Appendix VII. Relation of the R-F Load Voltage to the Power and VSWR in a Slotted Transmission Line}

Equation 9 is derived_as follows:

$$
\begin{aligned}
P_{R F} & =\frac{V_{\max } V_{\min }^{5}}{Z_{0}}, \\
\rho & =\frac{V_{\max }}{V_{\min }} .
\end{aligned}
$$

Substituting for $V_{\min }$ in eq 39 yields

$$
P_{R F}=\frac{V_{\max }^{2}}{\rho Z_{0}}
$$

Let

$$
\frac{V_{A}}{V_{\max }}=K_{1}
$$

${ }^{5}$ Principles of radar, by the staff of the MIT Radar School, pp. 8 to 45 (The Technology Press, Massachusetts Institute of Technology, Cambridge, Mass., 1944).
$=$ ratio of the voltage $\lambda / 2$ from the load to the maximum voltage in the line.

Then

and

$$
P_{R F}=\frac{V_{A}^{2}}{K_{1}^{2} \rho Z_{0}}
$$

$$
V_{A}=K_{1}\left(Z_{0} P_{R F} \rho\right)^{\frac{1}{2}}
$$

The ratio $V_{\max } / V_{A}$ was determined in two steps. First, when the line was loaded, the probe on the line was located where the voltage was a maximum. The probe output $D_{m}$ was recorded. The probe was then moved to the position corresponding to $\lambda / 2$ from the load, and the probe output $D_{A}$ was noted. The line was then shorted and the probe moved to where the voltage was a maximum in the line. The r-f level was adjusted until a $V_{S \max }$ corresponding to the maximum indication, $D_{m}$, was reproduced ( $V_{S}$ designates shorted line conditions). Then two positions were found on the line that produced the indication $D_{A}$, and the distance in centimeters between these points (both on the same side of the maximum) was recorded. This distance was called $\Delta_{S}$. The distance between a maximum and a minimum corresponded to $\lambda / 4(\pi / 2$ radians $)$. Since the voltage distribution in the line on short circuit was very nearly sinusoidal, $D_{A}=D_{M}$ $\cos \theta$, where $\theta$ was the angle in radians between $D_{M}$ and $D_{A}$. But $2 \theta / \pi=l /(\lambda / 4)$, where $l$ is the distance in centimeters from $V_{S \max }$ to $V_{A}$ on the line. $l$ also equaled $\left(\lambda / 4-\Delta_{S} / 2\right)$. Substituting and solving for $\theta$ yields $\theta=\pi\left(0.5-\Delta_{S} / \lambda\right)$. Therefore

$$
K_{1}=\frac{V_{A}}{V_{\max }}=\cos \theta=\cos \left(0.5-\frac{\Delta_{S}}{\lambda}\right) \pi .
$$

The VSWR is determined in a similar manner, i. e., by reproducing the probe outputs corresponding to $V_{\max }$ and $V_{\min }$ at two positions, one of them at a voltage maximum of the probe with the line shorted. The distance between these two positions in electrical degrees is then the angle, the cosine of which is equal to $\rho$. Sequence of steps suitable for various standing-wave ratios, as well as a general analysis of this method of measuring voltage ratios along a transmission line is presented by Winzemer. ${ }^{6}$

\footnotetext{
${ }^{6}$ A. M. Winzemer, Methed for obtaining the voltage standing-wave ratio on transmission lines independently of the detector characteristics. Thesis presented to the Polytechnic Institute of Brooklyn (May 1948).
}

\section{Washington, April 18, 1949.}

